\title{
Transforming growth factor- $\beta$ gene polymorphisms in different phenotypes of sarcoidosis
}

\author{
S. Pabst*, T. Fränken*, J. Schönau*, S. Stier ${ }^{\#}$, G. Nickenig*, R. Meyerף, \\ D. Skowasch* and C. Grohé ${ }^{+}$
}

ABSTRACT: The aetiology of sarcoidosis is unclear. Single nucleotide polymorphisms (SNPs) in transforming growth factor (TGF)- $\beta 2$ and $-\beta 3$ have been reported to be associated with the development of lung fibrosis in patients with sarcoidosis.

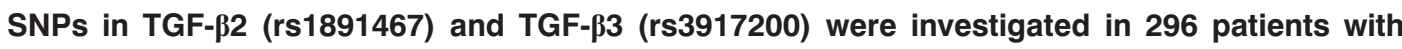
sarcoidosis (acute/self remitting, $n=70$ (including 62 patients with Löfgren's syndrome); chronic, $\mathrm{n}=168$; acute/chronic, $\mathrm{n}=58$ ) by real-time PCR. 32 patients showed radiological signs of lung fibrosis. The genotype frequencies were compared among the sarcoidosis groups as well as to 377 healthy controls.

We found a significant association with the G-allele in rs1891467 in TGF- $\beta 2$ and an acute/self remitting course of sarcoidosis compared to a chronic course $(p=0.001)$. The results were even more evident for patients with Löfgren's syndrome $(p<0.001)$. Moreover, we could demonstrate a borderline significance between TGF-p3 (rs3917200) and lung fibrosis $(p=0.050)$.

Carriers of the G-allele in rs1891467 might be protected from developing a chronic course. Moreover, there is evidence that rs3917200 is involved in the development of lung fibrosis in sarcoidosis. This study is the first in sarcoidosis patients to suggest a genetic implication of TGF$\beta 2$ as a protective factor in the course of sarcoidosis.

KEYWORDS: Genetics, genotype-phenotype association, lung fibrosis, sarcoidosis, transforming growth factor- $\beta$

S arcoidosis is an inflammatory granulomatous multisystem disorder, which in $>90 \%$ of cases primarily affects lungs and lymph nodes. Other organs, including skin, heart and liver may also be afflicted. The disease is characterised by noncaseating granulomas and an exaggerated cellular immune response caused by increased inflammatory activity of macrophages [1]. Chest radiography is used to describe the different stages of pulmonary sarcoidosis. The clinical course of most sarcoidosis patients is short and favourable, but about 20\% develop complications such as lung fibrosis, requiring long-term treatment with corticosteroids [2, 3].

Although considerable efforts towards understanding the pathogenesis of sarcoidosis have been made, the aetiology remains unclear. Evidence suggests that it is the product of an unknown exogenous antigenic stimulus and an endogenous genetic susceptibility [3]. A recently published registry-based twin study showed an 80 -fold increased risk of developing sarcoidosis in co-twins of affected monozygotic brothers or sisters compared with the general population [4]. The increased risk in dizygotic twins was sevenfold. This suggests that genetic impact in the aetiology of sarcoidosis is important. Moreover, there is evidence that distinct genes have the possibility to alter the course of the disease. KRUIT et al. [5] were able to show that single nucleotide polymorphisms (SNPs) in the transforming growth factor beta (TGF- $\beta$ ) gene isoforms are associated with the development of lung fibrosis in patients with pulmonary sarcoidosis. TGF- $\beta$ isoforms regulate components of the adaptive immune system, such as T-cells, and of the innate immune response, such as natural killer cells $[6,7]$. As there is evidence that SNPs in TGF- $\beta 2$ and $-\beta 3$ are associated in the development of lung fibrosis in patients with sarcoidosis, we attempted to analyse these results in a large Caucasian cohort of 296 German sarcoidosis patients with a mean follow-up time of 6.9 yrs. We hypothesised that SNPs in TGF- $\beta$ isoforms could be involved in the conversion to a chronic disease.

\section{AFFILIATIONS}

*Dept of Internal Medicine II,

Division of Pneumology,

\# Medizinische Poliklinik,

"Institute of Physiology II, University of Bonn, Bonn, and

${ }^{+}$Evangelische Lungenklinik Berlin Buch, Berlin, Germany.

CORRESPONDENCE

S. Pabst

Dept of Internal Medicine II, Division of Pneumology

University of Bonn

Sigmund-Freud-Str. 25

D-53105 Bonn

Germany

E-mail: stefan.pabst@ukb.uni-

bonn.de

Received:

July 282010

Accepted after revision: Nov 062010

First published online:

Dec 092010

European Respiratory Journal Print ISSN 0903-1936 Online ISSN 1399-3003 


\section{MATERIALS AND METHODS Patient population}

This study was in conformity with the Declaration of Helsinki (1989) of the World Medical Association, and was approved by the ethics committee of Bonn University School of Medicine, Bonn, Germany (number 080/05). Written informed consent was obtained from each patient and of all healthy controls prior to their enrolment.

Patients with severe medical disorders, including chronic obstructive lung disease, allergic asthma or immunological disorders, were excluded from the study. All patients were at least 18 yrs of age.

\section{Sarcoidosis group}

296 Caucasian patients with diagnosed sarcoidosis (age $53.0 \pm 12.9$ yrs; 168 females (56.8\%) and 128 (43.2\%) males) were included in the present study. They were recruited from the outpatient clinic of the Department of Internal Medicine, Rheinische-Friedrich-Wilhelms University, Bonn, Germany and from sarcoidosis peer-support groups in Wuppertal, Mainz and Cologne, Germany.

Sarcoidosis was confirmed by biopsy evidence of noncaseating epitheloid cell granulomas in any organ and chest radiograph (posterior-anterior and lateral) abnormalities. Chest radiographs were assessed in consensus by chest physicians and radiologists specialised in diffuse lung diseases to determine disease severity using standard radiographic staging for sarcoidosis, in accordance with American Thoracic Society (ATS)/European Respiratory Society (ERS)/World Association of Sarcoidosis and Other Granulomatous Disorders (WASOG) guidelines and the Scadding criteria: stage I characterises bilateral hilar lymphadenopathy, stage II additional parenchymal infiltrates, in stage III merely pulmonary infiltrates can be seen. Stage 0 describes a normal chest radiograph (including extrapulmonary manifestation), stage IV lung fibrosis [3, 8].

The diagnosis was further completed by history data, physical examination, lung function tests, chest computed tomography (81 patients), and bronchoalveolar lavage fluid analysis.

For the statistical analysis, patients with chronic course were defined as either 1) having symptoms over a time span of 2 yrs or more or 2) having had a minimum of two episodes in a lifetime, as described previously [9-11]. Acute sarcoidosis was defined as only one episode of a clinically acute sarcoidosis, which had totally resolved and had not relapsed in the follow-up time. Therefore, all patients with only one episode of Löfgren's syndrome and all patients with an otherwise acute form of sarcoidosis were included in this group, whereas patients with two or more episodes of Löfgren's syndrome or a chronic persistent or a relapsing course were grouped into the "chronic group". The mean follow-up time was $6.9 \mathrm{yrs}$ in all sarcoidosis patients (4.2 yrs in the "acute group"). Löfgren's syndrome was defined according to the ATS/ERS/WASOG guidelines [3]. 70 patients presented with an acute course, 62 of these suffered from Löfgren's syndrome. The remaining eight patients of the "acute group" did not show classical Löfgren's syndrome and had primarily skin and eye involvement. Lung fibrosis was diagnosed by chest radiography according to the Scadding criteria as described before (stable stage IV or progressive toward this stage) $[5,8]$. Accordingly, the non-fibrosis group comprises all patients with radiological stage 0 , I and II as well as two patients of stage III which presented with no signs of fibrosis in the most recent chest radiograph. The baseline characteristics of the sarcoidosis groups are shown in table 1.

\section{Control group}

377 Caucasian, healthy and unrelated volunteers who were age- and sex-matched served as the control cohort (age $53.2 \pm 17.6$ yrs; 202 (53.6\%) females and 175 (46.4\%) males). All were residents of Germany and were selected in preemployment examinations at the University of Bonn, Bonn, Germany. None had a history of lung disease or showed any symptoms of lung or other disease. All showed a normal chest radiograph and laboratory results, including complete blood count, urine analysis, hepatic enzyme activities and blood urea nitrogen levels.

\section{Methods}

Peripheral venous blood samples of $9 \mathrm{~mL}$ were drawn from each individual by standard venous puncture. DNA was purified from EDTA blood using the salting out protocol described by MiLLer et al. [12].

\section{SNP selection and genotyping}

SNPs rs1891467 (TGF- $\beta 2$ ) and rs3917200 (TGF- $\beta 3$ ) were selected as both were reported to be associated with lung fibrosis in sarcoidosis [5]. The SNPs, alleles and structures of PCR primers and fluorescence labelled detection probes for the variant TGF$\beta 2$ and - $\beta 3$ alleles are given in table 2 . For genotyping the TGF$\beta 2$ and $-\beta 3$ polymorphisms, real-time PCR analysis was used (Light-Cycler ${ }^{\mathrm{TM}}$; Roche, Mannheim, Germany), hybridisation probes were applied in combination with a Light-Cycler ${ }^{\mathrm{TM}}$ DNA Master Hybridization Probes Kit (Roche). Both the PCR primers and the fluorescence labelled detection probes were synthesised by TIB MolBiol, Berlin, Germany.

For PCR, conditions were $3 \mathrm{mM} \mathrm{MgCl}_{2}, 1 \mathrm{pmol}$ of each hybridisation probe, 20 pmol of the two PCR primers each, $2 \mu \mathrm{L}$ of Light-Cycler ${ }^{\mathrm{TM}}$ DNA Master Hybridisation Mix (Roche) and $100 \mathrm{pg}$ to $10 \mathrm{ng}$ DNA in a final volume of $20 \mu \mathrm{L}$. After $5 \mathrm{~min}$ of denaturation (at $95^{\circ} \mathrm{C}$ ), 40 PCR cycles (TGF- 33 ) and 45 PCR cycles (TGF- $\beta 2$ ) were performed with $3 \mathrm{~s}$ denaturation at $95^{\circ} \mathrm{C}$, $20 \mathrm{~s}$ annealing at $58^{\circ} \mathrm{C}$ (TGF- $\beta 3$ ) and $54^{\circ} \mathrm{C}$ (TGF- $\left.\beta 2\right)$ and $25 \mathrm{~s}$ extension at $72^{\circ} \mathrm{C}$. Differentiation of the TGF- $\beta 2$ and $-\beta 3$ alleles was performed by determination of melting curves after PCR. Melting curves were obtained following a denaturation period of $5 \mathrm{~s}$ at $95^{\circ} \mathrm{C}$ at a start temperature of $45^{\circ} \mathrm{C}$ and a final temperature of $80^{\circ} \mathrm{C}$, with a temperature gradient of $0.4^{\circ} \mathrm{C} \cdot \mathrm{s}^{-1}$. PCR and melting procedure were detected online with the Light-Cycler ${ }^{\mathrm{TM}}$ instrument. As an internal control, in 10 randomly chosen assays sequencing on an automated DNA sequencer was performed (Qiagen, Hilden, Germany), which was compared to the results by the Light-Cycler ${ }^{\mathrm{TM}}$ technique. For each sample, melting curves were produced, showing a temperature-dependent decrease in fluorescence intensity (figs 1 and 2). The melting curve analysis shows different melting maxima $(-\mathrm{dF} / \mathrm{dT})$ for the hybridisation probes, depending on the genotype. Concerning TGF- $\beta 2$, there was a single melting maximum of $58.0^{\circ} \mathrm{C}$ in the case of the wild-type and two melting maxima of $56.1^{\circ} \mathrm{C}$ and $64.2^{\circ} \mathrm{C}$ in heterozygous DNA. Homozygous variant genotypes showed a melting point of $56.1^{\circ} \mathrm{C}$. For TGF- $\beta 3$, a single melting 
TABLE 1 Baseline characteristics of sarcoidosis patients and healthy controls

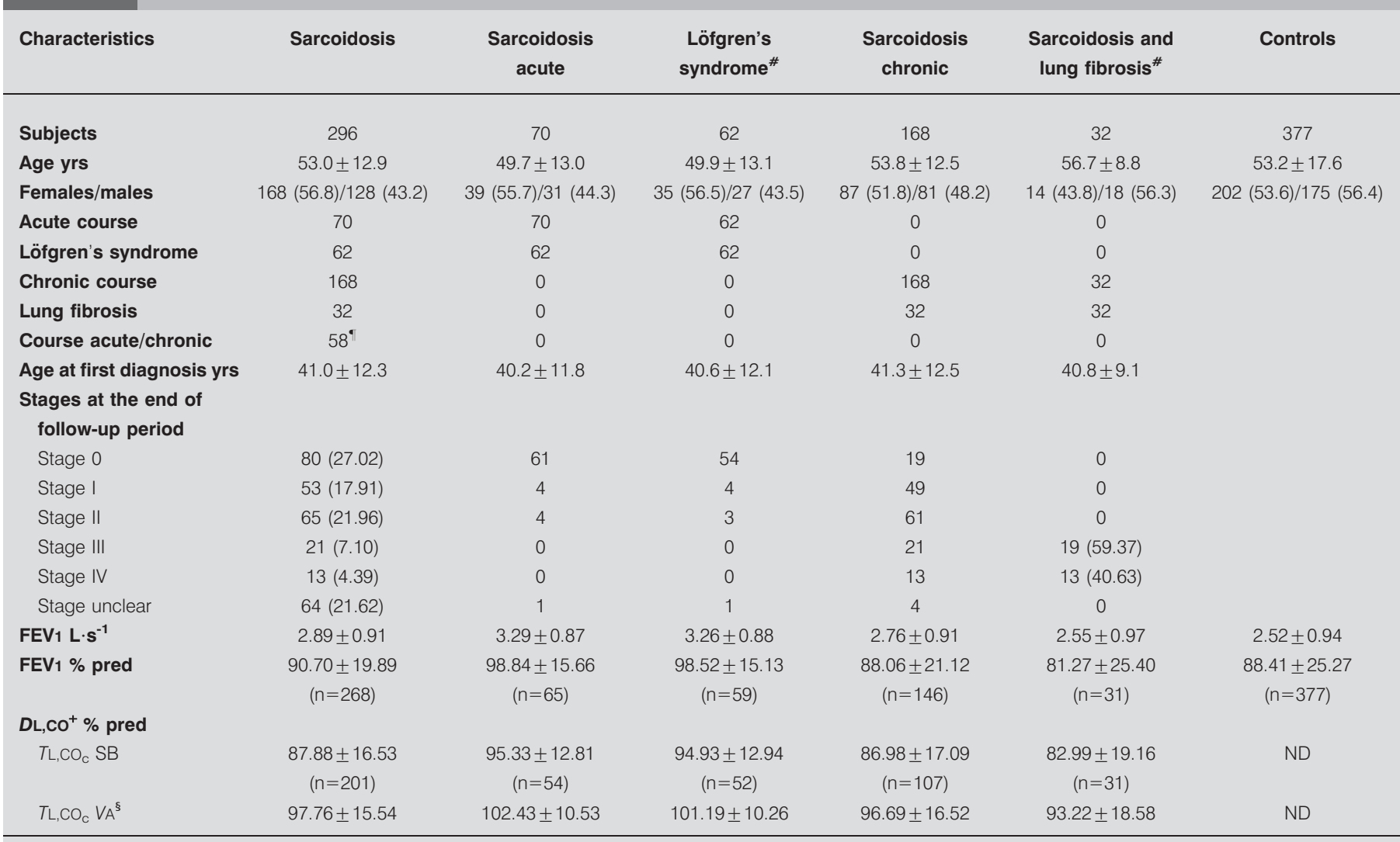

Data are presented as $n, n(\%)$ or mean $\pm \mathrm{SD}$, unless otherwise stated. FEV1: forced expiratory volume in $1 \mathrm{~s} ; \%$ pred: \% predicted; $D L, C O$ : diffusing capacity of the lung for carbon monoxide; $T \mathrm{~L}, \mathrm{CO}_{\mathrm{c}}$ : transfer factor of the lung for carbon monoxide, corrected for haemoglobin; $\mathrm{SB}$ : single breath technique; $\mathrm{VA}$ : alveolar volume; ND: not done. \#: all 62 patients with Löfgren's syndrome are part of the acute group, 32 patients with lung fibrosis are part of the chronic group; ": mean observation time 6.9 yrs, in 51 patients the course is either acute or chronic because the observation time was less than two years, and seven patients were lost in follow-up; ${ }^{+}$: data was available in $68.0 \%$ of all patients, $96.9 \%$ of patients with fibrosis, and was not performed in healthy controls; ${ }^{\varsigma}$ : Krogh factor.

maximum of $67.0^{\circ} \mathrm{C}$ was found in the case of the variant genotype and of $58.0^{\circ} \mathrm{C}$ in the case of reference genotype. In heterozygotes, the two melting maxima were located at $58.0^{\circ} \mathrm{C}$ and $67.0^{\circ} \mathrm{C}$, as shown in figures 1 and 2 .

Thus, the differentiation of genotypes was possible by melting curve analysis. For internal control, 10 randomly selected samples were sequenced and showed 100\% concordance with the Light-Cycler ${ }^{\mathrm{TM}}$ data.

\section{Statistical analysis}

Descriptive statistical analysis was performed using software SPSS (version 14.0, Chicago, IL, USA). Single marker association was evaluated with Pearson's Chi-squared test [13]. Genotype frequencies were tested for Hardy-Weinberg equilibrium (HWE). Statistical power was calculated with an online tool (case control for discrete traits test) [14].

Statistical significance was denoted at $\mathrm{p}<0.05$ for all tests performed.

\section{RESULTS}

To determine whether SNPs in the TGF- $\beta 2$ and $-\beta 3$ genes influence the susceptibility or the phenotype of sarcoidosis, we performed an association analysis of the markers in our control group against all sarcoidosis, acute course sarcoidosis, chronic course sarcoidosis, fibrosis and non-fibrosis sarcoidosis groups. The results are presented in table 3 . The distributions of allele carrier and genotype frequencies of TGF- $\beta 2$ and TGF$\beta 3$ in the sarcoidosis patients and healthy control subjects did not deviate from the Hardy-Weinberg equilibrium.

We compared patients with an acute course towards a chronic course of sarcoidosis and each group towards the control group. In TGF- $\beta 2$ we detected much less mutated G-allele in the cohort with a chronic course of sarcoidosis. We found significant associations of TGF- $\beta 2$ rs1891467: $p=0.001$ for chronic versus acute and $p=0.030$ for chronic versus controls. For the Löfgren's cohort, we could show the same results (Löfgren's versus chronic, $\mathrm{p}<0.001$ ). For TGF- $\beta 3$ we could not demonstrate significant results.

In a second calculation we compared 32 fibrotic with 200 nonfibrotic patients. 64 patients of the total sarcoidosis cohort had to be excluded as we could not obtain the current radiological stage. In TGF- $\beta 3$, we observed more patients with the mutated $\mathrm{C}$-allele in patients with pulmonary fibrosis with a p-value of 0.050 . In TGF- $\beta 2$, patients with fibrosis showed less mutated 


\begin{tabular}{|c|c|c|c|c|}
\hline TABLE 2 & $\begin{array}{l}\text { Primer } \varsigma \\
\text { (TGF)- } \beta\end{array}$ & $\begin{array}{l}\text { ces for the identification of biallelic single nucleotic } \\
\beta 3 \text { genes }\end{array}$ & ymorphisms in the & sforming growth fa \\
\hline Polymorphism & dbSNP & DNA sequence & Primers & Sensor/anchor \\
\hline $\begin{array}{l}\text { TGF- } \beta 2 \\
59941 \mathrm{~A} / \mathrm{G}\end{array}$ & rs1891467 & $\begin{array}{l}\text { 5' T TGT AAA CAG GCA ACT TAA ATA CAT CCT GAT GCC ATA } \\
\text { TGA ATA GTG GTA CTT GCA TAT AGG GTA TAG GCG GGG AAA } \\
\text { TIT CAC CAG GGA GCT GAC ATT TTG ATG AGG CCT TGA GAC } \\
\text { GTA TCT ATT AAA ACC TGA TGG GGG ATC ATC ATT CTT GGCA } \\
\text { GGA AGG GCA GGC ACT GCA AAG ACA GTC TTG AAT GGG } \\
\text { CTT GCT GAG GGT ACC TGA TGC ATA GCG CTC AGT GCC } \\
\text { TGG AGG TGA GGA GAG ACT GGG GAG AAG GTG GCC CTC } \\
\text { CAA AGA TAG TGT GTA GAG TGA CAC TAC AGA GGA TT '3 }\end{array}$ & $\begin{array}{l}\text { Forward primer: 5'GTA } \\
\text { AAC AGG CAA CTT } \\
\text { AAA TAC A '3 } \\
\text { reverse primer: 5'CAC } \\
\text { TCT ACA CAC TAT CTT } \\
\text { TGG A '3 }\end{array}$ & $\begin{array}{l}\text { Sensor A : 5'- GCT GAC ATT } \\
\text { TTG ATG AGG CC--FL'3 } \\
\text { anchor: 5'- LC640-TGA } \\
\text { GAC GTA TCT ATT AAA } \\
\text { ACC TGA TGG GGG-- } \\
\text { PH - '3 }\end{array}$ \\
\hline $\begin{array}{l}\text { TGF- } \beta 3 \\
17369 \text { T/C }\end{array}$ & rs3917200 & $\begin{array}{l}\text { 5' CA CAC CTC CCT CGC AGA CTG CAC TGC CCC TCC TCC } \\
\text { TGG GCA GTG ATG GGG CGT GTG GAG GAG GCA CCC TCC } \\
\text { AAG GGC TCT GCT CTC ITC AGA CAG GAG ATT GTC ACT TTC } \\
\text { CTT CCC TTC TTC AGG CGT GGA CAA TGA GGA TGA CCA TGG } \\
\text { CCG TGG A '3 }\end{array}$ & $\begin{array}{l}\text { Forward primer: 5' CAC } \\
\text { CTC CCT CGC AGA CT ' } 3 \\
\text { reverse primer: } 5 \text { ' CAT } \\
\text { GGT CAT CCT CAT } \\
\text { TGT CC ' } 3\end{array}$ & $\begin{array}{l}\text { Sensor: 5' - GCT CTG CTC } \\
\text { TCC TCA GAC AG--FL'3 } \\
\text { anchor: 5' - LC640- GAT } \\
\text { TGT CAC TTT CCT TCC } \\
\text { CTT CTT CAG GC--PH }\end{array}$ \\
\hline
\end{tabular}

The base exchanges are printed in bold. The sequence for the Light-Cycler sensor is shown in italics. The sequence for anchor-TGF- $\beta 2 / 3$ is printed in italics and underlined. The insertions for the primers are underlined. dbSNP: single nucleotide polymorphism database.

G-allele than patients without fibrosis with a clear statistical trend in Pearson's chi-square test $(\mathrm{p}=0.091)$.

\section{DISCUSSION}

We examined two SNPs in the TGF- $\beta 2$ and TGF- $\beta 3$ gene for the association with the course of sarcoidosis and with the development of lung fibrosis. The G-allele in rs1891467 seems to protect of developing a chronic course. Therefore, to the best of our knowledge this study is the first in sarcoidosis patients to suggest a genetic implication of TGF- $\beta 2$ as a potential protective factor in the course of sarcoidosis. Moreover, for both SNPs we could observe a clear, for TGF- $\beta 3$ even a borderline significant, trend towards the formation of lung fibrosis.

There is growing evidence that only genetically susceptible individuals will suffer from sarcoidosis. As the postulated

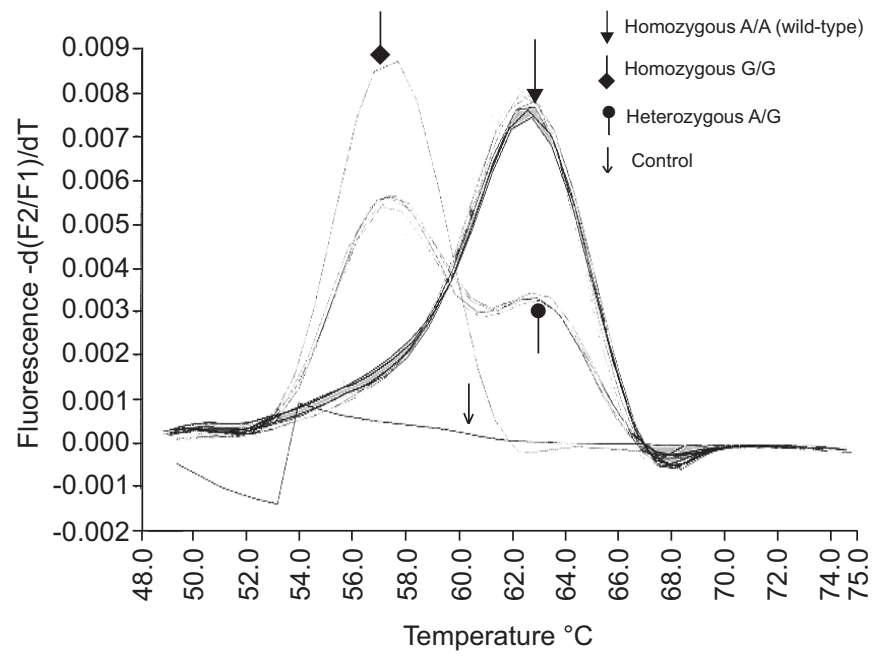

FIGURE 1. Melting curve of transforming growth factor- $\beta 259941$ A/G (rs1891467). exogenous stimulus leading to a sarcoidosis is still not found yet, a lot of effort has been undertaken in the last decade to find "the" sarcoidosis gene or a pattern of susceptibility genes that may lead to sarcoidosis. Furthermore the finding of disease modifying genes is of growing importance. These genes could be either protective, terminating the disease spontaneously like Löfgren's syndrome, or may alter the disease towards a chronic course with multiple organ involvement such as lung fibrosis.

Therefore in this study we wanted to answer the question if SNPs in TGF- $\beta 2$ (rs1891467) and - $\beta 3$ (rs3917200) may alter the course of sarcoidosis or might have a protective or harming influence towards the development of pulmonary fibrosis in patients with sarcoidosis. Both examined SNPs were chosen as there is a recent publication from KRUIT et al. [5], in which these

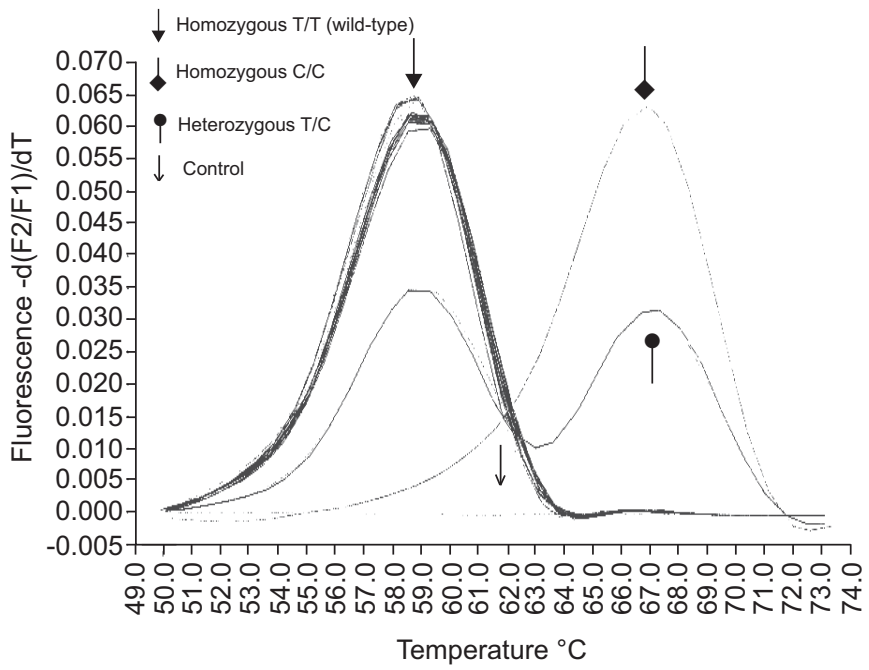

FIGURE 2. Melting curve of transforming growth factor- $\beta 317369$ T/C (rs3917200). 
TABLE 3 Statistical analysis of variants at the transforming growth factor (TGF)- $\beta 2$ and $-\beta 3$ loci

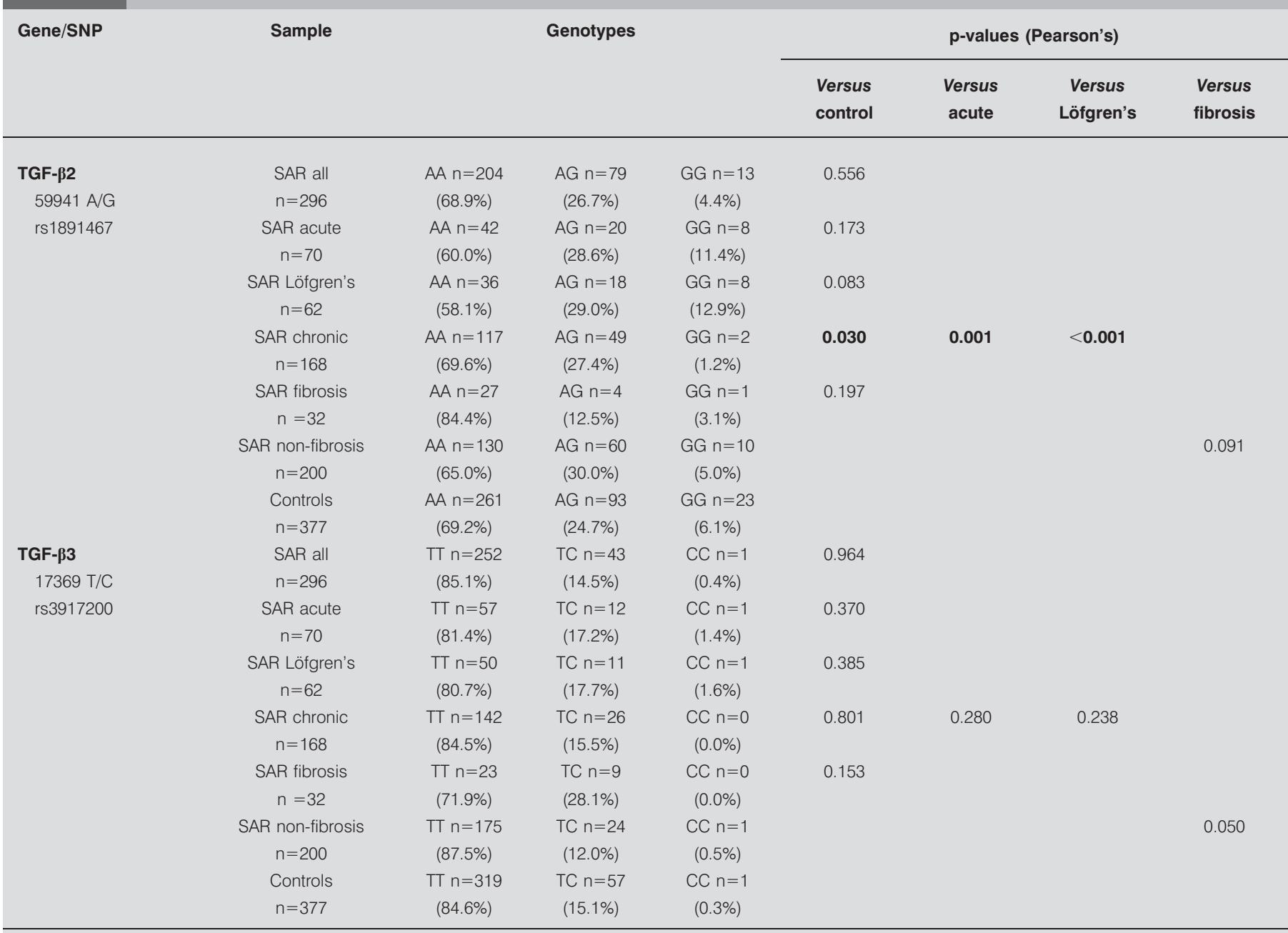

Significant associations are printed in bold. SNP: single nucleotide polymorphism; SAR: sarcoidosis; all: all patients.

SNPs were associated with a higher rate of pulmonary fibrosis in a Dutch cohort of patients with sarcoidosis.

The main finding of our study is the diminished amount of mutated G allele of TGF- $\beta 259941$ A/G in patients with a chronic course of sarcoidosis. Besides the fact that in the chronic group we found less wild-type A-allele, the main reason for the significant finding obviously is the lower number of patients with a homozygous G-mutation. Comparing the chronic to the fibrosis group, interestingly in the chronic group we observed more patients with homozygous mutated alleles. Actually we would have expected that fibrotic patients more frequently tend to a chronic course and, vice versa, chronic patients will develop more often a pulmonary fibrosis. The most probable reason for this discrepancy is that "chronic course" and "fibrosis" are two distinct phenotypes that might overlap in only some rare cases. Probably there is more than one genetic factor at play.

As mentioned above we were not able to replicate the results of the Dutch cohort in detail. Moreover, in TGF- $\beta 2$ we detected less mutated alleles in the fibrosis group, whereas KRUIT et al.
[5] found significantly more mutations in the fibrosis group. Our TGF- $\beta 3$ results and the results of the Dutch cohort were pointing into the same direction, with a borderline statistical significance in our cohort. These partially different results might be explainable due to the samples sizes of the fibrosis groups with 32 patients in our study and 24 in the Dutch study. The fibrosis patients of both studies were assigned to the fibrosis cohort based on the radiological findings and not the combination of lung function and radiological observations. We conclude that "lung fibrosis" sarcoidosis patients consist of a heterogeneous group of patients with a variable course determined by both genetic and clinical parameters. The genotype distribution in the control groups of both studies is quite similar for TGF- $\beta 317369 \mathrm{~T} / \mathrm{C}$. But there are evident differences in the genotype distribution of TGF-32 59941 A/G. Therefore, it might be possible to reach different results with an enlarged subcohort of fibrosis patients. We have to admit that in both studies, the Dutch and ours, the analysis of the small fibrosis groups are probably underpowered.

However, although functional data is still missing, we think that the TGF- $\beta$ family is a promising target in the genetics of 
pulmonary fibrosis in sarcoidosis. The crosstalk between TGF- $\beta$ isoforms is not fully understood. The three isoforms TGF- $\beta 1$, TGF- $\beta 2$ and TGF- $\beta 3$ have been associated with different functions [15]. Whereas the isoforms 1 and 2 of the TGF- $\beta$ family have been described generally with profibrotic properties, TGF- $\beta 3$ appears to have a more diverse nature [16, 17]. A recent study showed that TGF- $\beta 3$ might only have modulating effects on the expression of TGF- $\beta 1$ [18]. A previously published study was able to demonstrate that all three isoforms have the capability to force the synthesis of collagen by fibroblast stimulation and thus contribute to fibrosis. The different isoforms varied considerably in their potency. TGF- $\beta 3$ was 10 -fold more potent than the other two isoforms [19]. In another study of the same group, TGF- $\beta 3$ gene expression also was detected in spontaneous human pulmonary fibrosis [20]. Here, however, TGF- $\beta 1$ gene expression was highest. In a recent study, profibrotic effects in rat lungs could be detected after exogenous addition of TGF- $\beta 3$. Nevertheless, also in this case the induced profibrotic effects of TGF- $\beta 1$ predominated [18].

Concerning sarcoidosis, a study by Zissel et al. [21] found increased TGF- $\beta 1$ levels associated with a spontaneous remission. A recent study of the ACCESS group, exploring sarcoidosis susceptibility genes, was able to demonstrate associations of functional TGF- $\beta 1$ haplotypes and a chronic course of sarcoidosis [22]. It is not clear which functional effects are caused by the examined SNPs in our study. Therefore in future studies the function of the TGF- $\beta$ isoforms, especially TGF- $\beta 2$ and $-\beta 3$, and the functional influence of the SNPs lying in these genes are still to be explored.

The exact phenotyping makes or breaks the performance of genetic association studies in complex diseases, especially when performing analyses of subcohorts. In this study the definition of lung fibrosis is difficult. It is usual to classify sarcoidosis patients according to the conventionally radiological classification of SCADDING [8]. But nowadays, computed tomography (CT) often is performed in addition to chest radiography. Due to the enhanced resolution of CT scans, patients with sarcoidosis often are classified as radiological stage III or IV, even if no changes in conventional chest radiography are evident. We tried to rule this out by regarding only conventional chest radiography results, but a bias by knowing CT scan results might be present. Even though the fibrosis group presents with a lower transfer factor for carbon monoxide $(\mathrm{TL}, \mathrm{CO})$ in lung function testing not all patients with radiologically diagnosed lung fibroses show a functional impairment in TL,CO. Interestingly, $T \mathrm{~L}, \mathrm{CO}$ in this study is much higher than in the Dutch study (83 versus $63 \%$ ). However, these differences are very hard to interpret as, in our study, TL,CO data were available in 31 of 32 patients, whereas in the Dutch study the calculation was based on only seven of 23 patients with fibrosis. This makes a direct to comparison of both studies difficult.

Furthermore phenotyping in acute and chronic course sarcoidosis is not specific, but has proven to be a useful descriptive tool. This has been demonstrated for BTNL-2 (butyrophilinlike 2), which until now is the gene best associated with sarcoidosis and preferentially with a chronic course [10, 23]. The division into the two subgroups "acute" and "chronic" as we have defined it might have been arbitrary. Of course there are many other possible ways in which to phenotype sarcoidosis patients. In cohorts even larger than ours different phenotyping tools might be eligible. At the present time we would propose, for large cohorts, the SCAC (sarcoid clinical activity classification) phenotyping, as recently defined by PRASSE et al. [24]. This classification also makes a cut after 2 yrs to define a chronic course. Therefore we think our subclasses are reasonably chosen, and subdivision in the six SCAC classes would result in subcohorts too small for our study. Our sample size reached 296 patients, although sarcoidosis is a rare disease; therefore, this study should have enough statistical power to generate valid results. Compared to other genetic studies in sarcoidosis, 296 well phenotyped patients equals, or even exceeds, the number of subjects in previously published studies [25-27].

In summary, we examined two SNPs, one in the TGF- $\beta 2$ and on in the TGF- $\beta 3$ gene, for the association with the course of sarcoidosis and with the development of lung fibrosis. Carriers of the G-allele in rs1891467 in TGF- $\beta 2$ seem to be protected from developing a chronic course. This study is the first in sarcoidosis patients to suggest a genetic implication of TGF- $\beta 2$ as a protective factor in the course of sarcoidosis. Moreover, in both SNPs we could observe a clear, and for TGF- $\beta 3$ a nearly significant, trend towards the formation of lung fibrosis.

\section{SUPPORT STATEMENT}

This study was supported by institutional grants (BONFOR).

\section{STATEMENT OF INTEREST}

None declared.

\section{ACKNOWLEDGEMENTS}

We would like to thank all patients and healthy controls who made this study possible. Moreover we would like to thank H. and B. Stachetzki of the "sarcoidosis circle Bonn" for their help in implementing the "Sarcoidosis Network Bonn" (www.sarkoidose-bonn.de), which enabled the recruitment of the majority of patients for our project. Finally we would like to thank M. Lennarz (University of Bonn, Bonn, Germany) for her excellent technical support in conducting the experiments.

\section{REFERENCES}

1 Iannuzzi MC, Rybicki BA, Teirstein AS. Sarcoidosis. N Engl J Med 2007; 357: 2153-2165.

2 Hunninghake GW, Crystal RG. Pulmonary sarcoidosis: a disorder mediated by excess helper T-lymphocyte activity at sites of disease activity. N Engl J Med 1981; 305: 429-434.

3 Statement on sarcoidosis. Joint Statement of the American Thoracic Society (ATS), the European Respiratory Society (ERS) and the World Association of Sarcoidosis and Other Granulomatous Disorders (WASOG) adopted by the ATS Board of Directors and by the ERS Executive Committee, February 1999. Am J Respir Crit Care Med 1999; 160: 736-755.

4 Sverrild A, Backer V, Kyvik KO, et al. Heredity in sarcoidosis - a registry-based twin study. Thorax 2008; 63: 894-896.

5 Kruit A, Grutters JC, Ruven HJ, et al. Transforming growth factor- $\beta$ gene polymorphisms in sarcoidosis patients with and without fibrosis. Chest 2006; 129: 1584-1591.

6 Laouar Y, Sutterwala FS, Gorelik L, et al. Transforming growth factor- $\beta$ controls $\mathrm{T}$ helper type 1 cell development through regulation of natural killer cell interferon- $\gamma$. Nat Immunol 2005; 6: 600-607. 
7 Li MO, Wan YY, Sanjabi S, et al. Transforming growth factor- $\beta$ regulation of immune responses. Annu Rev Immunol 2006; 24: 99-146.

8 Scadding JG. Prognosis of intrathoracic sarcoidosis in England: a review of 136 cases after 5 years' observation. BMJ 1961; 2: 1165-1172.

9 Li Y, Wollnik B, Pabst S, et al. BTNL2 gene variant and sarcoidosis. Thorax 2006; 61: 273-274.

10 Pabst S, Karpushova A, Diaz-Lacava A, et al. VEGF gene haplotypes are associated with sarcoidosis. Chest 2010; 137: 156-163.

11 Pabst S, Baumgarten G, Stremmel A, et al. Toll-like receptor (TLR) 4 polymorphisms are associated with a chronic course of sarcoidosis. Clin Exp Immunol 2006; 143: 420-426.

12 Miller SA, Dykes DD, Polesky HF. A simple salting out procedure for extracting DNA from human nucleated cells. Nucl Acids Res 1988; 16: 1215.

13 Plackett RL. Karl Pearson and the Chi-squared test. Int Stat Rev 1983; 51: 59-72.

14 Purcell S, Cherny SS, Sham PC. Genetic Power Calculator: design of linkage and association genetic mapping studies of complex traits. Bioinformatics 2003; 19: 149-150.

15 Awad MR, El-Gamel A, Hasleton P, et al. Genotypic variation in the tranforming growth factor- $\beta 1$ gene: association with transforming growth factor $\beta 1$ production, fibrotic lung disease, and graft fibrosis after lung transplantation. Transplantation 1998; 66: 1014-1020.

16 Nath RK, LaRegina $\mathrm{M}$, Markham $\mathrm{H}$, et al. The expression of transforming growth factor type $\beta$ in fetal and adult rabbit skin wounds. J Pediatr Surg 1994; 29: 416-421.

17 Shah M, Foreman DM, Ferguson MW. Neutralisation of TGF- $\beta 1$ and TGF- $\beta 2$ or exogenous addition of TGF- $\beta 3$ to cutaneous rat wounds reduces scarring. J Cell Sci 1995; 108: 985-1002.
18 Ask K, Bonniaud P, Maass K, et al. Progressive pulmonary fibrosis is mediated by TGF- $\beta$ isoform 1 but not TGF- $\beta 3$. Int J Biochem Cell Biol 2008; 40: 484-495.

19 Coker RK, Laurent GJ, Shahzeidi S, et al. Transforming growth factors- $\beta 1,-\beta 2$, and $-\beta 3$ stimulate fibroblast procollagen production in vitro but are differentially expressed during bleomycininduced lung fibrosis. Am J Pathol 1997; 150: 981-991.

20 Coker RK, Laurent GJ, Jeffery PK, et al. Localisation of tranforming growth factor- $\beta 1$ and $\beta 3$ mRNA transcripts in normal and fibrotic human lung. Thorax 2001; 56: 549-556.

21 Zissel G, Homolka J, Schlaak J, et al. Anti-inflammatory cytokine release by alveolar macrophages in pulmonary sarcoidosis. Am J Respir Crit Care Med 1996; 154: 713-719.

22 Jonth AC, Silveira L, Fingerlin TE, et al. TGF- $\beta 1$ variants in chronic beryllium disease and sarcoidosis. J Immunol 2007; 179: 4255-4262.

23 Valentonyte R, Hampe J, Huse K, et al. Sarcoidosis is associated with a truncating splice site mutation in BTNL2. Nat Genet 2005; 37: 357-364.

24 Prasse A, Katic C, Germann M, et al. Phenotyping sarcoidosis from a pulmonary perspective. Am J Respir Crit Care Med 2008; 177: 330-336.

25 Franke A, Fischer A, Nothnagel M, et al. Genome-wide association analysis in sarcoidosis and Crohn's disease unravels a common susceptibility locus on 10p12.2. Gastroenterology 2008; 135: 1207-1215.

26 Mrazek F, Kvezereli M, Garr E, et al. Complement receptor 1 single nucleotide polymorphisms in Czech and Dutch patients with sarcoidosis. Tissue Antigens 2008; 71: 77-80.

27 Spagnolo P, Sato H, Grunewald J, et al. A common haplotype of the C-C chemokine receptor 2 gene and HLA-DRB1*0301 are independent genetic risk factors for Löfgren's syndrome. J Intern Med 2008; 264: 433-441. 